\title{
Arabia Saudí y las dinámicas de financiarización en Oriente Medio: algunas repercusiones geopolíticas del proyecto Neom en el Mar Rojo MoIsés GARDUÑo GaRCía*
}

\begin{abstract}
RESUMEN
Este artículo estudia el papel del proyecto Neom en las dinámicas de financiarización de Arabia Saudí en Oriente Medio y expone algunas de sus repercusiones regionales. El texto describe a Neom como un proyecto impulsado por élites globales transnacionales el cual implica, para llevarse a cabo, una serie de maniobras militares por parte de Riad en el Mar Rojo en conjunto con la ayuda de países aliados en la región como Egipto e Israel. El artículo se divide en tres partes: la primera expone el marco teórico ofrecido por autores como Saskia Sassen,William Robinson y David Harvey en el que se utiliza la relación entre financiarización, nuevo urbanismo y militarismo para explicar el fenómeno a estudiar. La segunda parte explica la inserción de algunas élites saudíes en las élites globales transnacionales para entender el papel que desempeña Neom en la conducta saudí en el Mar Rojo. Finalmente, la última parte analiza algunas consecuencias regionales del fenómeno, enfatizando en las oportunidades y obstáculos que tienen los saudíes en la zona del Mar Rojo para crear un nuevo balance de poder político y económico en dicha área.
\end{abstract}

\section{Palabras clave}

Financiarización; Neom; élites globales transnacionales; Mar Rojo

\section{Tithe}

Saudi financialization dynamics in the Middle East: some geopolitical consequences of Neom's project in the Red Sea

\section{Abstract}

This paper addresses the role of Neom's project in the Saudi dynamics of financialization in the Middle East and exposes some of its regional repercussions in the Red Sea area. The text describes Neom as a project promoted by global transnational elites which needs a series of military maneuvers between Riyadh and its allies in the region, especially Egypt and Israel.The article is divided into three parts: the first one exposes the relationship between financialization, new urbanism and militarism as theoretical framework to explain the phenomenon. The second part explains the insertion of some Saudi elites in transnational global elites to understand the role that Neom is playing in Saudi behavior in the Red Sea. Finally, the last part analyzes some regional consequences of this policy, emphasizing the opportunities and obstacles in the Red Sea for Saudis and its allies.

\section{KEYWORDS}

Financialization; Neom; global transnational elites; Red Sea.

Este artículo se enmarca en los trabajos del PAPIIT IN305I I 9 Sectarismo y justicia social en el Medio Oriente del siglo XXI apoyado por la DGAPA de la UNAM. Un agradecimiento especial se manifiesta para el Grupo Coimbra por el apoyo brindado durante una estancia de investigación en el verano de 2019 en la Universidad de Salamanca, España, donde este trabajo fue terminado.

\section{DOI:}

http://doi.org//0.15366/relacionesinternacionales2019.42.008

Formato de citación recomendado:

GARDUÑO GARCÍA, Moisés. "Arabia Saudí y las dinámicas de financiarización en Oriente Medio: algunas repercusiones geopolíticas del proyecto Neom en el Mar Rojo”, en Relaciones Internacionales, n 42, 2019, pp. I4I-I56.
*Moisés GARDUÑO GARCÍA, Licenciado en Relaciones Internacionales por la UNAM, Maestro en Estudios de Asia y África con especialidad en Medio Oriente por El Colegio de México y Doctor en Estudios Árabes e Islámicos Contemporáneos por la Universidad Autónoma de Madrid. Actualmente es profesor de Tiempo Completo en la Facultad de Ciencias Políticas y Sociales de la UNAM donde dirige el Taller de Estudios sobre Medio Oriente. También coordina el Módulo de Medio Oriente dentro del Diplomado en Estudios sobre Asia del Seminario de Estudios Asiáticos de la UNAM.

Recibido: 08/03/2019

Aceptado: 09/09/2019 
A una década de la crisis económica de 2008, la financiarización sigue provocando un gran interés en los Estudios Internacionales ya que se trata de una etapa del sistema capitalista donde, después de las fases mercantil, industrial y (neo)liberal, la brecha entre ricos y pobres se ha acrecentado con gran velocidad en todo el mundo. De acuerdo con el reporte Public Good or Private Wealth, de la organización no gubernamental Oxfam, en enero de 2019 la cantidad de personas multimillonarias se duplicó al pasar de I I 25 personas en 2008 a 2208 en el 2018'. Nombres como Jeff Bezos de Amazon, Bill Gates de Microsoft, Warren Buffett de Berkshire Hathaway o Mark Zuckerberg de Facebook encabezan la lista de las veintiséis personas cuya riqueza alcanzó cerca de I,4 trillones de dólares, una suma equiparable a la riqueza total de los 3800 millones de personas más pobres del planeta.

El capitalismo financiero se produce en el marco de sociedades posindustriales, es decir, un espacio de reproducción hegemónica cuya principal característica es la centralidad de los mercados financieros y la especulación ${ }^{2}$. Las sociedades posindustriales viven en red, en un contexto cibercultural y con una dispersión geográfica de las actividades económicas donde el conocimiento es uno de los elementos más valiosos para los actores transnacionales y los gobiernos que conviven con ellos. La ciudad, en este orden de ideas, es el espacio por excelencia para la réplica de este modelo 3 .

Lo anterior implica el reconocimiento de una crisis de la ciudad como espacio social. Esto es ocasionado por la contradicción entre el capitalismo financiero como modelo, por un lado, y la fragmentación social causada por la gentrificación, por el otro. Una evidencia de esto es la convivencia entre las zonas residenciales - hogar de clases acomodadas- y las llamadas ciudades perdidas — hogar de los desposeídos -; fenómeno que demuestra que el término ciudad global se encuentra lejos de cumplir con las promesas de desarrollo generalizado - tal como se concibió- y se encuentra, por el contrario, reflejando la compleja relación que existe entre la polarización, racismo, desigualdad, clasismo y aporofobia que se produce cuando se contraponen ambas narrativas en un mismo espacio ${ }^{4}$.

Hablar de un nuevo urbanismo no implica referirse sólo a aquellos paisajes basados en rascacielos, centros comerciales y todo un rediseño de la movilidad multimodal, sino en pensar sobre las nuevas interacciones que esta infraestructura produce en un contexto informático donde existen personas que pueden tener acceso a las dinámicas de producción, distribución y consumo y aquellas que no lo tienen ${ }^{5}$. Para complementar esta idea, Gustavo Lins Ribeiro propone hablar de la transición a una nueva era tecnológica que denomina "capitalismo comunicacional” la cual está produciendo dinámicas como el trabajo en casa, la alta productividad, el outsourcing y una

\footnotetext{
OXFAM, "Public Good or Private Wealth, Universal health, education and other public services reduce the gap between rich and poor, and between women and men. Fairer taxation of the wealthiest can help pay for them", Oxfam International, Oxford. p. I2. Disponible en: https:// www.oxfamamerica.org/static/media/files/bp-public-good-or-private-wealth-210II9-en.pdf [consultado: 2 de febrero de 2019].

2 BELL, Daniel, The Coming of Post-Industrial Society:A Venture in Social Forecasting. Basic Books, New York, 1973.

3 En la década de los noventa, Saskia Sassen ya había advertido sobre esto. SASSEN, Saskia, The Global City: New York, London, Tokyo, Princeton University Press, Oxford, I99I, p.6.

4 SASSEN, Saskia, “La ciudad global. Una introducción al concepto y a su historia”, en Brown Journal of World Affairs, vol. I I (2), I 995. Pp. 27-43.

5 GARDUÑO, Moisés. “Medio Oriente: demografía, migración y derechos humanos a siete años de las revoluciones árabes”, en BALLESTEROS, Carlos (coord.). Las Regiones Internacionales, FCPYS-UNAM, México, 2019, p. 88.
} 
exacerbación de la competencia liberal y el narcicismo que reproduce nuevas lógicas de usufructo de mano de obra. Tomando en cuenta lo que el taylorismo, el fordismo y el toyotismo representaron para el capitalismo industrial, el googleísmo es para Lins Ribeiro el concepto que mejor representa las interacciones del capitalismo financiero, particularmente cuando ya no sólo se trata de la explotación laboral en el sentido estricto del fenómeno, sino de una forma de autoexplotación laboral ${ }^{6}$.

Lo anterior pone de manifiesto la graduación de una nueva élite al frente de este proyecto global ${ }^{7}$. Una nueva clase capitalista transnacional que surgió como un segmento de la burguesía mundial y que representa a los nuevos propietarios de este modelo de ciudad y la programación de sus algoritmos ${ }^{8}$. Así, grandes corporaciones que se aprovechan de estas nuevas condiciones participan cada vez más en sectores estratégicos para la vida de millones de personas tales como la agroindustria, las maquiladoras, el turismo transnacional, la importación de remesas relacionada con el fenómeno de la migración internacional y, por supuesto, la información estratégica para la generación de tecnología de alta gama donde la inteligencia artificial y la nanotecnología tiene un papel importante'. La plusvalía generada por el capital de estas corporaciones es usada para construir otras ciudades globales donde se reproduce el modelo, por lo que en una lógica compartida por David Harvey y David Rothkopf se puede decir que las nuevas ciudades están diseñadas para los intereses del capital y no para los ciudadanos, una especie de Tierra de ricos (richistan) ${ }^{10}$ :

"Business leaders in Buenos Aires, Frankfurt, Hong Kong, Johannesburg, Istanbul, Los Angeles, Mexico City, Moscow, New Delhi, New York, Paris, Rome, Seoul, Singapore, and Tokyo all read the same newspapers, wear the same suits, drive the same cars, eat the same food, fly the same airlines, stay in the same hotels, and listen to the same music. [This integrated elite also includes] international bureaucrats ... [who] coordinate policy... on global issues such as trade, the environment, health, development, and crisis management ${ }^{1 !} . "$

Conectado con lo anterior, es necesario mencionar la relación con el militarismo. El papel del armamento es vital para la recolección de la plusvalía y para la construcción de nuevos espacios. Ya David Harvey ha examinado el papel que desempeña la guerra en el capitalismo cuando la reflexiona como una forma de acumulación en sí misma ${ }^{12}$. $Y$ es que el militarismo puede construir la percepción de una amenaza, bombardear una ciudad y, posteriormente, reconstruirla durante la etapa posconflicto, creando una nueva lógica territorial que reproduzca los valores e

\footnotetext{
6 LINS, Gustavo. “El precio de la palabra: la hegemonía del capitalismo electrónico-informático y el googleísmo”, en Desacatos, 58, 20I8, pp. 16-33. Para otros autores se trata de un pro-sumidor, una persona que, al consumir, produce el rastro de sus gustos. BUENO, Carmen, "Innovación abierta: de consumidores a productores de valor", en Desacatos, 58, 2018, pp. 50-69.

7 HARRIS, Jerry. “Globalization, technology and the transnational capitalist class”, Foresight. I7, 20I5, pp. $194-207$.

8 ROBINSON, William. Latin America and global capitalism: a critical globalization perspective, Johns Hopkins University Press, Baltimore, 20 I0, p. 25.

9 GARCIA, Néstor, “Como investigar la era comunicacional del capitalismo”, en Desacatos, 56, 20I8, pp. 90 - I05.

10 HARVEY, David. "The right to the City”, en David Harvey Personal Web Page. Disponible en: https://davidharvey.org/media/righttothecity.pdf [consultado: 3 de febrero de 2019]

"Citado en ROBINSON William, Idem.

12 HARVEY, David, A Brief History of Neoliberalism. Oxford University Press, Cary, p. I 59.
} 
ideologías de los vencedores tal como ocurrió en la invasión a Iraq de $2003^{13}$. Esta técnica, en los últimos cincuenta años, ha ayudado a dinamizar el sector de la construcción, eje central y pilar de la financiarización. En la época actual, las empresas de seguridad están muy relacionadas con las tecnologías de la información tal como lo muestra el hecho de que de las veinticinco compañías de ciberseguridad más ricas del mundo en el año 2018 cinco sean empresas estadounidenses productoras de armas, es decir, Raytheon Co., Lockheed Martin Corp., BAE Systems, Booz Allen Hamilton y Northrop Grumman Corp ${ }^{14}$.

\section{Arabia Saudí y el capitalismo financiero: petróleo y urbanismo}

De acuerdo con Michael Field, los Estados árabes modernos emergen de una cultura donde los comerciantes eran autoridades que amasaron fortunas de la recolección de perlas y el impuesto a caravanas de provincias vecinas ${ }^{15}$. En particular, en los Estados del Golfo las principales empresas se crearon con base en fuertes vínculos familiares asociados tanto al gobierno de sus respectivos Estados, como al petróleo que soportaría el gran peso de su funcionamiento institucional ${ }^{16}$. Si bien el inicio del modelo capitalista industrial terminó con el modelo tradicional de economía en la región —así como con la industria naval árabe-, comenzó su integración a la economía capitalista, primero a través de negocios y posteriormente con la explotación de los energéticos.

A diferencia de otras monarquías donde el gobierno se instalaba en un Estado preexistente, las familias del Golfo crearon sus propios Estados mediante tres alianzas: una entre la familia Al Saud y las tribus locales que produjeron un sistema de lealtad, una segunda entre los Al Saud y los gremios AIWahab de La Meca y Medina que vivían de la renta de la peregrinación, y, en tercer lugar, otra alianza con grandes mercaderes de Yidda, en el Hiyaz ${ }^{17}$.

Lo anterior dio pie a la existencia de una red de élites políticas, militares, religiosas y comerciales donde los descendientes de Ibn Saud se encargarían de los asuntos principales del Estado, la seguridad y la economía, mientras los descendientes de AIWahab, se encargarían de los asuntos religiosos, la justicia y la educación. Por su parte, las élites comerciales se crearon a partir de la incorporación de las familias adineradas como figuras subsidiarias, clientes y proveedoras de un Estado que, desde su creación, subsistió con un modelo rentista, pues el petróleo solo profundizó aquel sistema ya existente, lo que permitió una relación entre la élite gobernante y los comerciantes basada en matrimonios, la provisión de bienes y servicios a la sociedad, y la fabricación de una clase contratista que ha dependido del Estado, no sólo para su desarrollo interno, sino para su proyección al exterior. Familias como los Mandil, los Nafisi, los Faisán y los Gosaybi representan una evidencia de este argumento ${ }^{18}$.

\footnotetext{
13 EGAN, Daniel. "Globalization and the invasion of Iraq State power and the enforcement of neoliberalism", en CARLTON-FORD, Steven \& ENDER, Morten (ed.) The Routledge Handbook of War and Society. Iraq and Afghanistan, Routledge, London, p. 189.

14 KELLER, John, "Five U.S defense contractors rank among the world's top 25 cyber security companies", en Military \& Space. Disponible en: https://www.militaryaerospace.com/articles/20I8/06/cyber-security-trusted-computing-defense-contractors.html [consultado: 4 de febrero de 2019].

15 FIELD, Michael, The Merchants:The Big Business Families of Saudi Arabia and the Gulf States, Overlook Press, Woodstock, N.Y. I985, p. 3 I4.

${ }^{16}$ En el caso de Arabia Saudí, la Casa de Alireza (Beit Alireza) fundada en 1845 por Zainal Alireza, es un claro ejemplo de cómo grandes familias y confederaciones tribales llegaron a convertirse en poderosas clases transnacionales de la mano de sus interlocutores extranjeros como Ford Motors.Ver HAJJI ABDULAH ALIREZA \& CO., LTD. “Alireza”. Disponible en: http://www.alireza.com/ [consultado: 20 de febrero de 20I9].

17 SOLER I LECHA, Eduard y Luciano Zaccara, “Arabia Saudí familia, religión, ejército y petróleo”, en BRICHS, Ferran Izquierdo (coord.) Poder y regímenes en el mundo árabe contemporáneo, CIDOB, Barcelona, 2009, p. 232.

18 BASSENS, David, BEN Derudder yWITLOX, Frank, "Searching for the Mecca of finance: Islamic financial services and the world city network",
} 
Pero ha sido la presencia del petróleo lo que en verdad facilitó que las empresas transnacionales se instalaran en la región mientras el capital de las familias árabes se instalaba en las ciudades globales del mundo mediante el papel fundamental de los fondos soberanos, esto es, fondos de inversión propiedad del gobierno saudí con fines macroeconómicos en sectores estratégicos como el acero, el oro, instancias bancarias como Soft Bank, entre otros, cuya aplicación está limitada a la decisión última de la familia real. Por ejemplo, esto se puede rastrear en compañías como Uber, o en la refinería más grande de Estados Unidos en Puerto Arturo; aunque también se podrán encontrar petrodólares saudíes en Tesla, en compañías chinas en Liaoning y Zhejiang, o en proyectos de electricidad en Maharashtra, India, Johor, Malaysia o en Gwadar, Pakistan ${ }^{19}$. Otra fuente de transnacionalización del capital saudí son los servicios financieros islámicos, donde participan decenas de firmas con participación en 64 ciudades globales como Manama, Dubai y Abu Dhabi, asociadas con los servicios administrativos de la peregrinación por parte de bancos islámicos, asociaciones de caridad y fundaciones encargadas de manejar los impuestos de los fieles ${ }^{20}$.

Otra evidencia de la transnacionalización de los países árabes del Golfo es su injerencia en instituciones internacionales. El hecho que todos los miembros del CCG sean miembros de la Organización Mundial del Comercio (con Qatar siendo el anfitrión de la Ronda de Doha), es una evidencia para dicho argumento. En el caso particular de Arabia Saudí, el hecho que sea un país miembro de la Cámara de Comercio Internacional (ICC), de la Organización Internacional de Normalización (IOS) y de la Organización Mundial de Aduanas (OMA), es un elemento que ayuda a explicar la facilitación de iniciativas como la Zona de Libre Comercio de Oriente Medio (MEFTA) en conjunto con Estados Unidos, lo que ha acercado al reino a la lógica de las compañías transnacionales productoras de armamento, compras que sólo son superadas por las ventas del petróleo y que suelen ser interpretadas como el catalizador de desastres, intervenciones y crisis en países vecinos donde intervienen grandes complejos industriales-militares. De hecho, la antes mencionada invasión a Iraq en 2003 es la evidencia para este argumento, pues implicó una inversión de 607 mil millones de dólares entre 2002 y 2007 donde la participación de los Estados del Golfo fue central para llevar a cabo el teatro de operaciones tal como lo muestra la participación de compañías como Kuwaiti Trading and Contracting, Alargan Trading of Kuwait, Gulf Catering, y en el caso particular del reino, la Saudi Trading and Construction Company ${ }^{21}$.

Por otro lado, una evidencia más de la conexión saudí con las élites globales de la financiarización se puede ver a manera individual. De acuerdo con la revista Forbes,Arabia Saudí cuenta actualmente con siete multimillonarios y es el hogar del hombre más rico de la región, el Príncipe Alwaleed Bin Talal Alsaud, cuyo patrimonio ascendía, en 2017, a veinte mil millones de dólares con inversiones en compañías como News Corp, Citigroup, Four Seasons Hotels \& Resorts Twitter, el Hotel George V en París y el Savoy Hotel en Londres. En diciembre del año 20 I6, Alwaleed se unió a la revolucionaria Coalición Energética de Bill Gates con la promesa de una inversión de cincuenta millones de dólares ${ }^{22}$. Otra persona similar es Mohammed Al Amoudi, de origen etíope,

en Area,Vol. 42, No. I, 201 0, pp. 35-46.

19 MANJOO, Farhad, “Saudi Money Fuels the Tech Industry It's time to Ask Why”, en The New York Times, 6 de noviembre de 20I7: https://www. nytimes.com/2017/I I/06/technology/unsavory-sources-money-fueling-tech.html [consultado: 3 de marzo de 2019].

20 BASSENS, David, BEN Derudder yWITLOX, Frank, "Searching for the Mecca of finance: Islamic financial services and the world city network", en Area,Vol. 42, No. I, 2010 , pp. 35-46.

21 ROBINSON,William, Op. Cit. p. 237.

${ }^{22}$ FORBES. "Prince Alwaleed Bin Talal Alsaud”, en Forbes, 20 de marzo de 2017: https://www.forbes.com/profile/prince-alwaleed-bin-talal- 
pero con ciudadanía saudí quien hizo su fortuna invirtiendo internacionalmente, primero en Suecia y luego a través de una cartera diversificada en Etiopía y Arabia Saudí al exportar granos de café a Starbucks y hojas de té a Lipton, además de poseer refinerías de petróleo en Marruecos y Suecia y campos petrolíferos frente a las costas de África Occidental ${ }^{23}$.

Con respecto al urbanismo que sigue el modelo de financiarización, es necesario mirar al interior del reino. Aunque la torre más alta de Arabia Saudí (Abraj Al Bait) se encuentra en La Meca, la ciudad más importante en estos términos es la capital, Riad. Esta ciudad es considerada como más próspera del país con un PIB per cápita de aproximadamente treinta y dos mil dólares. Similar a lo que ocurre con otras ciudades globales de la zona como Dubai, Doha, Abu Dhabi - Muscat, la economía de Riad se basa principalmente en el auge del sector de la construcción impulsado con el dinero del petróleo. En un texto de Ángeles Espinosa, se reporta que este auge ha estado acompañado de profundos cambios sociales que están tratando de diluir una interpretación puritana del islam donde los saudíes tengan acceso a conciertos y espectáculos de artistas internacionales, un paisaje urbano con mujeres en bicicleta y eventos deportivos como la reciente carrera de Fórmula I, e incluso, un ministerio de la diversión ${ }^{24}$. Entre los proyectos más importantes en el ámbito doméstico se encuentran el metro de Riad, la ciudad Qidiyya, la Torre de Yedda, el complejo de Saudi-Aramco totalmente renovado, las avenidas nuevas de la capital, las ciudades para las fuerzas armadas, la planta solar de la King Abdullah Economic City y, por supuesto, Neom, en la cual se profundizará más adelante.

Pero el avance del modelo urbano saudí coexiste con la emergencia de una serie de problemáticas que representan un reto a esta conexión del reino con el capitalismo global. Una nueva generación de saudíes que cuenta con sus propias expectativas, con discursos críticos al régimen y con intelectuales perseguidos por la justicia como los del movimiento Sahwa ${ }^{25}$. El malestar de una población hiper-segregada constituye otro de los problemas, particularmente la discriminación contra población migrante pakistaní en barrios de Riad como Batha, Olaya, Nazeem y Jaradiah, producto del programa de centralización estatal que dio origen a circuitos-miseria, un efecto inevitable de la financiarización ${ }^{26}$. Otro problema es que los trabajadores perciben que pronto se enfrentarán a la ola tecnológica de la nueva generación que les sustituirá de algunas actividades que desempeñan hoy en día, desde la conducción de taxis hasta la repartición de mensajería ${ }^{27}$.

Finalmente, pero no menos importante, está el problema entre las mismas élites saudíes donde existe una fuerte oposición al Príncipe heredero, Mohammad Bin Salman, por sus polémicas decisiones que han conllevado a reprimir fuertemente a grupos disidentes tal como lo

alsaud/\#44c082a4I dbf [consultado: 20 de julio de 2019].

${ }_{23}$ ROBEHMED, Natali, "Meet the Richest Peoplein the Middle East", en Forbes, 26 de marzo de 2014: https://www.forbes.com/sites/ natalierobehmed/20 14/03/26/meet-the-richest-people-in-the-middle-east/\#39b794f2 Ibf9 [consultado: 20 de febrero de 20I9].

24 ESPINOSA, Ángeles, “Los saudíes abrazan el cambio sin cuestionar el orden establecido”, en El País, 5 de marzo de 20I9: https://elpais. com/internacional/20I9/03/0I/actualidad/I55|436078_66/407.html?id_externo_rsoc=FB_CC\&fbclid=IwAR0vdUdZV23SjTC dIIWIHleX2W26ctav9EzTv9BE7mjBcMQU6aKAIYOe0 [consultado: 6 de marzo de 2019].

${ }_{25}$ IBRAHIM,Arwa. "What is Sahwa, the Awakening movement under pressure in Saudi?, en Al Jazeera, 5 de junio de 20I9: https://www.aljazeera. com/indepth/features/sahwa-awakening-movement-pressure-saudi-I90603 I00555782.html [consultado: 6 de julio de 2019].

26 DAVIS, Mike., \& AMOROTO SALIDO, José María, Planeta de ciudades miseria, Foca, Tres Cantos, 2007.

27 MIRTAHERI, Seyed Ahmad, Transnational Capitalism and the Middle East: Understanding the Transnational Elites of the Gulf Cooperation Council, Thesis dissertation, Florida International University, 2016, pp. 39-42. 
demostraron el caso de Yamal Khashoqgi, por un lado, y la pugna con sus primos a quien encarceló en noviembre de 2017, por el otro, incluido el multimillonario Alwaleed Bin Talal por supuestos cargos de corrupción. Tal como lo reporta The New York Times, con esos arrestos Mohammed Bin Salman parece haberse hecho con el control de los tres servicios de seguridad saudíes más importantes: el ejército, los servicios de seguridad interna y la guardia nacional, durante décadas distribuidos entre las ramas del clan de la casa de Al Saud para conservar el equilibrio del poder en el país, lo que es señal de una clara confrontación entre sus intereses particulares y los de la "vieja guardia" a la que pertenecen Alwaleed Bin Talal, Mutaib Bin Abdallah y Abdel Fakeih, entre otros $^{28}$

Una evidencia para argumentar el descontento al interior del reino radica en las declaraciones de Saad Al Faqih, una prominente figura de oposición, quien ha denunciado desde Londres lo que él llama el autoritarismo represor de Bin Salman, su dependencia con la administración de Trump y el Estado de Israel, y sobre todo, la falta de un proyecto de nación que concilie la reforma con la tradición, llegando a considerar dos escenarios que podrían resultar en una revuelta social contra su proyecto:"la primera sería que los eventos regionales se intensificaran, llevando a una guerra con Irán, mientras la otra sería la derrota en la intervención en Yemen la cual conduciría al colapso de su poder y un punto de partida para unir jóvenes, trabajadores y líderes islamistas para comenzar una revuelta"29.

\section{El proyecto Neom en la agenda saudí 2030 y sus repercusiones regionales}

En este orden de ideas Mohammed Bin Salman presentó el modelo de desarrollo Vision 2030 que intenta acelerar la lógica del capitalismo financiero y que implica, entre otras cosas, una nueva ciudad global con base en tres pilares fundamentales: el estatus de Arabia Saudí como el corazón de los mundos árabe e islámico, la determinación de convertir al país en una potencia de inversión global, y la relevancia de su ubicación estratégica, la cual se considera única por ser un centro global que conecta tres continentes, Asia, Europa y África ${ }^{30}$.

Una de las razones de este plan es el interés saudí en dejar de depender de la explotación petrolera e invertir en el sector turístico por medio del desarrollo de ciudades con la más alta tecnología que puedan garantizar la reproducción del enorme capital del reino. La construcción de infraestructura en puertos, trenes y aeropuertos que garanticen que los musulmanes de todo el mundo puedan visitar los sitios sagrados del islam, la transformación de la empresa estatal Aramco en un conglomerado industrial global, el aumento de su Fondo de Inversión Pública para que se convierta en el fondo de riqueza soberana más grande del mundo y el fortalecimiento de su ejército para mantener la seguridad de su país son partes sustanciales de este plan ${ }^{31}$. Para

\footnotetext{
${ }^{28}$ KIRKPATRICK, David. “La purga del príncipe heredero y la transformación radical de Arabia Saudita”, en The New York Times en español, 8 de noviembre de 2017: https://www.nytimes.com/es/2017/II/08/purga-transformacion-arabia-saudita-mohamed-bin-salman/ [consultado el I9 de julio de 2019]

29 MIDDLE EAST MONITOR."Prominent Saudi opposition figure says a revolt is waiting to happen”, en Middle East Monitor, 19 de julio de 2019 https://www.middleeastmonitor.com/20 I 907/9-prominent-saudi-opposition-figure-says-a-revolt-is-waiting-to-happen/?fbclid=IwAR I nO7BJu 6PWj3C9afLyHjjzESfqKK2bfXE2-WVDDGUCqFhvq66o5Px2-Pw [consultado: 20 de julio de 20I9].

30 VISION 2030 KINGDOM OF SAUDI ARABIA. “Foreword”. Disponible en: https://vision2030.gov.sa/en/foreword [consultado el 3 de marzo de 2019]

${ }^{31}$ Los proyectos incluyen la expansión del Aeropuerto Internacional Rey Abdulaziz por 7200 mdd en Yida, el desarrollo del Puerto Rey Abdalá por 5000 mdd cerca del valle industrial de la Ciudad Económica del Rey Abdalá y los planes de la Dirección General de Inversiones de Saudi Arabia General Investment Authority, SAGIA) de invertir más de I4I 000 mdd en proyectos de ferrocarriles, metro y autobuses para 2024. ABDUL LATIF JAMEEL, “Arabia Saudí ¿está lista para liderar el desarrollo de ciudades inteligentes en el mundo?, en ALJ, I2 de agosto de 20 I8:
} 
esto, el reino intenta no sólo invertir en las compañías transnacionales, sino atraer inversión de ellas para comprometerlas en el desarrollo nacional una vez que Arabia Saudí abrió oficialmente su mercado a la inversión extranjera desde el año 201532. Este modelo atenta directamente con la vieja guardia saudí que se oponía a abrir su mercado a la inversión extranjera y, sobre todo, a las reformas sociales que dicha apertura implicaba, particularmente en lo concerniente a la dependencia de flujos extranjeros.

No obstante, en este marco Bin Salman presentó "la ciudad del futuro" o Neom ${ }^{33}$, la cual contempla la construcción de una megaciudad con jurisdicción propia - donde pueda consumirse alcohol y otras cosas normalmente prohibidas en el reino- de $26500 \mathrm{~km} 2$ en Tabuk, la zona fronteriza con Egipto y Jordania, en un espacio que pueda integrarse a un corredor turistico con Jerusalén, la Peninsula del Sinaí y Petra. Neom se presenta como una ciudad global que además pueda capitalizar todo el potencial que ofrece la más avanzada tecnología del mundo para abordar problemas asociados con la sostenibilidad, la congestión, el transporte y el uso de energía ${ }^{34}$.

Se planea que la primera fase de construcción del Neom Bay se lleve a cabo entre el primer trimestre de 2019 y 2020, previendo completarla en entre siete y diez años ${ }^{35}$. Este proyecto incluirá oportundades para conectar otras ciudades globales, aeropuertos, un puerto marítimo, zonas turísticas, complejos industriales y "centros de innovación" ${ }^{36}$. Asimismo este complejo tendrá la capacidad para desarrollar dieciséis sectores: energía, agua, movilidad, biotecnología, alimentos, manufactura, medios de comunicación, entretenimiento, cultura y moda, tecnología y digital, turismo, deporte, diseño y construcción, sector de servicios, salud, educación y habitabilidad ${ }^{37}$.

Para la construcción de Neom se tiene previsto un costo de más de 500 mil millones de dólares (provenientes del Fondo de Inversión Pública y de inversionistas internacionales), para lo cual, el gobierno del reino creó la Neom Company, misma que es propiedad del Fondo de Inversión Pública ${ }^{38}$ y que tendrá como objetivo supervisar los gastos de construcción de la megaciudad y su desarrollo. Aunque aún no hay información suficiente sobre los contratistas que participarán en el proyecto, algunas figuras de la élite global han manifestado el interés de aportar inversiones, entre ellos Tim Brown — presidente de IDEO_, Timothy Collins — fundador de Ripplewood Advisors—, Alexandra Cousteau —asesora principal de Oceana—, Dan Doctoroff —fundador y

https://www.alj.com/es/perspective/arabia-saudi-esta-lista-para-liderar-el-desarrollo-de-ciudades-inteligentes-en-el-mundo/ [consultado: 4 de marzo de 2019]

32 REUTERS, “Saudi Arabia to open stock market to foreigners on June 15”, en Reuters I6 de abril de 20I5: https://www.reuters.com/article/ saudi-bourse-investment/saudi-arabia-to-open-stock-market-to-foreigners-on-june-I 5-idUSL5N0XD35P20I 504 I 6 [consultado el 20 de julio de 2019]

${ }_{33}$ Neom proviene del prefijo en latín neo, que significa "nuevo”, mientras que la "M" refiere a la palabra árabe "Mostaqbal”, es decir,"futuro”,"la ciudad del futuro".

${ }^{34}$ De acuerdo con el Banco Mundial, este tipo de ciudades contempla el uso de redes móviles $5 G$, que se espera que sean hasta 60 veces más rápidas que las redes $4 \mathrm{G}$, el blockchain, inteligencia artificial y vehículos autónomos. La investigación ha sugerido que los taxis autónomos y los rápidos sistemas ferroviarios interurbanos podrían combinarse para reducir el número de automóviles hasta en un $90 \%$.Ver WORLD BANK, “Introduction to Smart cities”. Disponible en: https://olc.worldbank.org/content/introduction-smart-cities [consultado: 5 de marzo de 2019]

35 ARAB NEWS, “Saudi Arabia to start first phase of Neom Project”,Arab News. 16 de enero de 2019: http://www.arabnews.com/\%20node\%20 1\%20|436436\%20/\%20business-economy [Consultado: 27 de febrero de 20I9]

36 ARAB NEWS, "Saudi Arabia announces creation of Neom Company", en Arab News, 5 de febrero de 2019: http://www.arabnews.com/ node/I44389//saudi-arabia [Consultado: 26 de febrero de 2019]

37 NEOM, Foundations. https://www.neom.com/ [Consultado: 26 de febrero de 2019]

38 Idem. 
CEO de Sidewalk Labs_-, Masayoshi Son —presidente y CEO de SoftBank Group Corp-, Neeli Kroes —antigua Vicepresidenta de la Comisión Europea- ${ }^{39}$. De hecho, a pesar de lo ocurrido con el caso Khassoggi, Arabia Saudí celebró el evento Davos in the Desert con el cual intentó atraer a más compañías para inmiscurise en el proyecto. Lo cierto es que compañías ligadas a Alwaleed Bin Talal cancelaron su participación, principalmente Uber, mientras los actores aliados a a Bin Salman como Steve Mnuchim, Secretario del Tesoro estadounidense, y Christine Lagarde, entonces al frente del Fondo Monetario Internacional, atendieron al evento, en lo que demuestra que la Vision 2030, y el proyecto Neom en particular, son un intento por parte de Mohammad Bin Salman para subirse al modelo de la financiarización para competir con Alwaleed Bin Talal, utilizando su influencia política y su cercanía con la administración actual de Donald Trump.

Lo expuesto tiene profundas implicaciones en el nivel regional pues echar a andar este proyecto ha implicado mover sensibles piezas geopolíticas no sólo en el Golfo Pérsico tal como lo ha constatado el distanciamiento reciente con Qatar, sino sobre todo en la zona del Mar Rojo donde la guerra en Yemen es parte de esta ecuación.Y es que el nuevo urbanismo de Neom tendría una ubicación geoestratégica muy atractiva, pero también profundas consecuencias geopolíticas dado el rediseño subregional que considera la conexión del Canal de Suez - Mar Mediterráneo con el sur de África y el sub continente Indio a través del reforzamiento de la seguridad del Golfo de Aqaba y del estrecho de Bab Al Mandab.

Lo anterior debe tomarse en cuenta para analizar la intervención militar saudí en Yemen impulsada por Mohammed Bin Salman, la cual tiene una relación fundamental con el anhelo saudí de tener un mayor control sobre el tránsito de buques en el Golfo de Aqaba y en el Mar Rojo, sobre todo si se considera a Neom y al Mar Rojo como una ruta marítima que forma parte el proyecto de la Nueva Ruta de la Seda de China, lo que lo convierte no sólo en una ruta de tránsito, sino también en una ruta de producción, distribución y consumo potencial. La apertura de la base militar de China en Djibouti es una evidencia para este argumento, pues es del interés de Beijing aumentar la conectividad regional con el Mar Rojo para el paso de las mercancías que produce ${ }^{40}$. Otra evidencia para este argumento radica en el papel chino con respecto a la intervención saudí en Yemen, la cual no ha afectado las relaciones entre China y Arabia Saudí pese a la violación de derechos humanos y las múltiples críticas internacionales recibidas por Riad ante la crisis humanitaria. De hecho, la respuesta de China a los ataques aéreos dirigidos por Arabia Saudí, que fueron apoyados militarmente por Estados Unidos, fue silencio puro. Si bien, China no apoyó tales acciones militares, tampoco las condenó. ${ }^{41}$ Esto debe conectarse con el beneplácito saudí de las políticas chinas contra musulmanes uigures en Xijiang donde Mohammed Bin Salman no fue crítico con las políticas chinas de segregación y discriminación lideradas por el presidente chino ${ }^{42}$.

\footnotetext{
39 Por otra parte, Klaus Kleinfeld, quien fue CEO de Arconic, fue anunciado como director inaugural del proyecto Neom por Mohammed Bin Salman en 2017 y en julio de 2018 lo nombró su asesor, sucediéndole el cargo de director de Neom a Nadhmi AI-Nasr.ARAB NEWS, Klaus Kleinfeld named adviser to Saudi crown prince, Neom appoints new CEO, 3 de julio de 20 I 8: http://www.arabnews.com/node/I 33228I/saudi-arabia [Consultado: 27 de febrero de 2019]; Neom, The members of Neom. Disponible en: https://www.neom.com/ [Consultado: 27 de febrero de 2019].

40 DOMINGUEZ, Gabriela, “The Silk Road in the Red Sea”, en Canadian Center for Strategic Studies. Disponible en: https://ccss-cces.com/sections/ analysis/2018/05/the-silk-road-in-the-red-sea/ [consultado: 5 de marzo de 2019].

4I CHANG, Jennifer, "China and Yemen's forgotten War”, en United States Institute for Peace. Disponible en: https://www.usip.org/sites/default/ files/2018-0I/pb24I-china-and-yemens-forgotten-war.pdf [consultado: 5 de marzo de 2019].

${ }^{42}$ AL JAZEERA, "Saudi Crown Prince defends Chine's Right to Fight Terrorism”, en Al Jazeera, 23 de febrero de 20I9: https://www.aljazeera.
} 
Conectado con lo anterior, el apoyo de Estados Unidos y Reino Unido a la aventura militar saudí en Yemen se relaciona con la venta de armamento. De acuerdo con el Instituto Internacional de Estudios para la Paz de Estocolmo (SIPRI, por sus siglas en inglés), del año 2013 al año 2017 Arabia Saudí fue el primer cliente de armas estadounidense y británicas al comprar el $18 \%$ y el $49 \%$ respectivamente del total de dichas exportaciones ${ }^{43}$. Esto se traduce en que, en el mismo periodo de tiempo,Arabia Saudí se convirtió en el segundo mayor importador de grandes armas del mundo al incrementar sus importaciones en un $225 \%$ en comparación con el periodo 2008-12, mismo ejercicio que se manifestó con sus principales aliados en la región, Egipto (que fue el tercer mayor importador del mundo entre 2013-17) al crecer un 215\% entre 2008-12 y 2013-17, y Emiratos Árabes Unidos que fue el cuarto mayor importador en 2013-17 del mundo en el mismo periodo ${ }^{44}$. Lo anterior se tradujo en ganancias millonarias para las compañías estadounidenses y británica que lideran la lista de las cien compañías más ricas del ámbito armamentista, específicamente Lockheed Martin, Raytheon Company, BAE Systems, Northrop Grumman, Boeing y General Dynamics ${ }^{45}$.

Por otro lado, este proyecto ha llevado a Arabia Saudí a buscar una alianza con Egipto e Israel, países considerados clave para el éxito de Neom. En el caso de El Cairo se trata de un aliado clave en tanto Neom necesita mano de obra para ser construida, necesita la herencia egipcia como atracción principal de turistas a nivel mundial $y$, particularmente, necesita infraestructura para mejorar la seguridad de las fronteras. Egipto ha mantenido históricamente números elevados de migrantes en la Península Arábiga que trabajan en el sector energético, lo que había sido una válvula de escape a la crisis de empleo juvenil por la que atravesó el país del Nilo durante la primera década del siglo XXI la cual llegó al $35 \%$ en el $2013^{46}$. Estratégicamente, Neom proyecta la buena salud de las relaciones entre ambos países pues la donación de las Islas de Tirán y Sanafir por parte de Al Sisi a Riad en junio de 2017 pasa por esta lógica al ser parte del proyecto para la construcción del Puente King Abdel Aziz, a cargo de The Bin Laden Group, el cual unirá a Arabia Saudí con Egipto a través de dichas islas a pesar de múltiples protestas contra el presidente egipcio ahora habilitado para gobernar hasta el año $2030^{47}$.

En este mismo tenor, es preciso recordar que Arabia Saudí apoyó a Al Sisi cuando el gobierno estuvo a cargo de la Hermandad Musulmana, sobre todo con dinero que iría a parar a programas sociales para contener la fuerza de la juventud egipcia ${ }^{48}$. Tan solo en el paquete inicial, apenas derrocado Mohammed Morsi, Arabia Saudí contribuyó con un paquete de ayuda de cinco mil millones de dólares, incluyendo mil millones en efectivo, dos mil millones en suministros

com/news/2019/02/saudi-crown-prince-defends-china-fight-terrorism-190223 I04647|49.html [consultado el 6 de marzo de 2019.

43 SIPRI. "Trends in International Arms Transfers, 20I7” Marzo de 20I8: https://www.sipri.org/sites/default/files/20I8-03/fssipri_at20I7_0.pdf [consultado: 4 de marzo de 2019].

44 lbídem.

45 DEFENSE NEWS, “Top ten for 2018”. Disponible en: https://people.defensenews.com/top-100/ [consultado: 4 de marzo de 2019].

46 GHAFAR, Adel Abdel, “Educated but unemployed: the challange facing Egypt youth”, en Arab Reform Initiative, octubre 20I7: file:///Users/ moisesgarduno/Downloads/Adel\%20Abdel\%20Ghafar_0.pdf [consultado: 4 de marzo de 2019].

47 THE NEW YORK TIMES, “Egytian PresidentAgrees to Cede 2 Islands to Saudi Arabia”, en The New York Times, 24 de junio de 20 I7: https:// www.nytimes.com/2017/06/24/world/middleeast/egypt-abdel-fattah-el-sisi-tiran-sanafir.html [consultado: 3 de marzo de 20I9].

48 RASHED, Ingi, “NEOM, a new channelfor Egyptian-Saudi Cooperation”, en Egypt Today, I6 de agosto de 20I8: http://www.egypttoday.com/ Article/2/56096/NEOM-A-new-channel-for-Egyptian-Saudi-cooperation [consultado: 5 de marzo de 2019]. 
energéticos y otros dos mil millones en depósitos para el Banco Central Egipcio ${ }^{49}$.

Lo anterior invita a pensar que las élites gobernantes cercanas a Mohammed Bin Salman, conectadas con la financiarización, perciben a un Egipto aliado como un actor central para su seguridad y estabilidad no sólo en el Golfo Pérsico, sino también en el Mar Rojo.Por su parte, Egipto se ha vuelto cada vez más dependiente de las naciones ricas en petróleo del Golfo para mantener a flote su economía, relanzar el crecimiento económico tan lastimado en los últimos quince años y solucionar sus dificultades crónicas para equilibrar los pagos de créditos en el exterior. Lo que se quiere decir con esto, es que el panorama económico que implica Neom ha provocado un caldo de cultivo propicio para la creación de un acuerdo de seguridad sostenible entre ambos países que comparten narrativas tales como "la lucha contra la hermandad musulmana" o la "amenaza iraní". La evidencia para este argumento radica en que el dinero que Egipto recibió entre 2013 y 2015 por parte de Arabia Saudí se utilizó no sólo para apoyar los problemas sociales y económicos, sino también para financiar la compra de armas de Francia y Rusia, esto cuando Estados Unidos suspendió la ayuda militar a Egipto luego de la toma de control militar en julio de 2013. Al mismo tiempo, las remesas de los trabajadores continuaron llegando al país del Nilo a pesar de que los precios internacionales del petróleo se desplomaron en el verano de 2014 , lo que habla de la continuidad laboral que Riad otorgó exclusivamente a los ciudadanos egipcios. En 2015, Egipto recibió cerca de diecinueve mil millones de dólares en remesas, principalmente de los países del CCG50.

En el mismo ámbito regional, Arabia Saudí parece contar con los egipcios para el impulso de cualquier normalización de las relaciones entre Israel y el mundo árabe a través de un acuerdo de seguridad regional que contrarreste a Irán. Tel Aviv es también un actor importante en la conformación de una superclase global en la economía financiera y en el impulso de las ciudades globales, sobre todo en el ámbito de la seguridad con empresas como Checkpoint Software, Beni Tal, Private Security Guards o Cisco que monitorean los barrios y centros comerciales mejor acomodados del mundo, las bolsas de valores más importantes (particularmenteWall Street) y, algo muy importante, que cuenta con enorme influencia en los consorcios de comunicación de masas y de poder militar-industrial donde el papel del AIPAC en Washington, por ejemplo, es fundamental ${ }^{51}$. La evidencia para este argumento radica en que, tras la controvertida desaparición del periodista delWashington PostYamal Khashoggi, funcionarios del gobierno israelí como Benjamin Netaniahu y del gobierno estadounidense como Yared Kuhsner no se atrevieron a criticar abiertamente a su aliado político y defendieron, por el contrario, que "el gran enemigo para ellos siempre sería Irán". En este sentido, es menester mencionar que existen notas que sugieren cierta injerencia israelí en aquel polémico suceso ${ }^{52}$.

49 SAID, Abdel Monem y Hussein Ibish, Egypt-GCC Partnership: Bedrock of Regional Security Despite Fissures, The Arab Gulf States Institute in Washington, 12 de diciembre del 2016: https://agsiw.org/egypt-gcc-partnership-bedrock-regional-security-despite-fissures/ [consultado: 5 de marzo de 2019]

50 ADLY,Amr, “Will saudi-egyptian geopolitical partnership push for economic integration? increasing interdependencies”, en European University Institute, 2 de febrero de 2019: http://cadmus.eui.eu/bitstream/handle//8|4/6 I I25/MED_2019_02.pdf?sequence= I\&isAllowed=y [consultado: 5 de marzo de 2019].

51 GARDUÑO, Moisés (Coord.) Pensar Palestina desde el Sur Global, UNAM, México, 20 I7, p.338. En el caso de los medios de comunicación, ver MEARSHEIMER, John y WALT Stephen (2006). “The Israel lobby and U.S. foreignpolicy”, en Middle East Policy, XIII (3), p. 45.

52 WINSTANLEY, Asa, "The disappearance of Jamal Khashoggi and the Israel-Saudi Alliance", en Middle East Monitor, II de octubre de 20I8: https://www.middleeastmonitor.com/20I8I0II-the-disappearance-of-jamal-khashoggi-and-the-israel-saudi-alliance/ [consultado: 4 de marzo de 2019]. 
Para efectos del corredor turístico de Neom, Israel está muy interesado en apoyar el proyecto siempre y cuando se mantenga la narrativa iranofóbica que, de cierta manera, es el combustible que alimenta dicha relación geopolítica. Incluso, existen reportes en periódicos israelíes que defienden que el traspaso de las islas de Tirán y Tanafir de Egipto a Arabia Saudí pasó por los servicios de inteligencia israelíes para obtener el visto bueno de Tel Aviv antes de haberse dado a conocer públicamente ${ }^{53}$.

No obstante, una de las razones más poderosas por las que Israel ha respaldado el programa político y geopolítico saudí, incluyendo la posibilidad de un programa nuclear tal como lo han demostrado diversas fuentes estadounidenses, es que dicha expansión alimenta la rivalidad saudí-iraní no sólo en el Golfo Pérsico, sino ahora también en la zona del Mar Rojo ${ }^{54}$. Lo anterior, considera el mantenimiento de un estado de guerra permanente a la sombra de la proliferación nuclear, lo que es útil para la industria armamentista global, particularmente para la estadounidense y la israelí mientras, en palabras de Hamid Dabashi,"se mantiene a la población de ambos países preocupada por el imaginado conflicto entre ambas naciones, lo que hace a la audiencia de la esfera pública global cada vez más ajena a la difícil situación del pueblo palestino" "55. Un factor adicional es que, al tiempo de escribir estas notas, la aspiración nuclear de Arabia Saudí debe relacionarse con el hallazgo de "enormes reservas de gas natural en el Mar Rojo", lo que aumenta no sólo el potencial energético del país, sino su capacidad de financiar sus planes nucleares y la seguritización de los mismos ${ }^{56}$. A propósito de la seguritización, la evidencia más clara de esto es la firma de la llamada Alianza contra el Terrorismo (ETIDAL, por sus siglas en árabe) en diciembre de 2015, donde Abdel Fatah Al-Sisi dio nuevas señales para continuar desarrollando una relación bilateral con Arabia Saudí para la defensa de lo que él denominó la "seguridad nacional árabe" ${ }^{7}$.

Lo anterior ha provocado discrepancias con los Estados competidores de Arabia Saudí en el Golfo Pérsico, naciones cuyas élites también están impulsando el modelo de ciudad global y de financiación económica. El caso más paradigmático es el diferendo que existe entre Arabia Saudí y Qatar como consecuencia de la negativa qatarí de apoyar las decisiones de Riad al interior del CCG, además de cuestiones relacionadas con el ya mencionado apoyo de Doha a la Hermandad Musulmana en Egipto. Formalmente, Arabia Saudí acusó a Doha de "apoyar el terrorismo" en 2017. De hecho, impuso un bloqueo económico que fue apoyado por Bahréin, Emiratos Árabes Unidos y Egipto quienes además suspendieron los lazos diplomáticos con Qatar hasta que éste país cumpliera una serie de demandas entre las que se encuentran el cierre de la cadena de

53 THE TIMES OF ISRAEL, “Leaks showEgypt consulted Israel over transfer of Red Sea Islands”, en The Times of Israel, I I de febrero de 20I7: https://www.timesofisrael.com/leaked-recordings-show-egypt-consulted-israel-over-transfer-of-red-sea-islands/ [consultado: 5 de marzo de 2019].

54 FANDOS, Nicholas y MAZZETTI, Mark, "House Opens Inquiry Into Proposed U.S. Nuclear Venture in Saudi Arabia”, en The New York Times, 19 de febrero de 2019: https://www.nytimes.com/2019/02//9/us/politics/trump-saudi-arabia-nuclear-power.html [consultado: 7 de marzo de 2019].

55 DABASHI, Hamid, “The making of a nuclear MBS”, en Al Jazeera, 7 de marzo de 2019: https://www.aljazeera.com/indepth/opinion/makingnuclear-mbs-190307| 35 I |4759.html?fbclid=IwARIHm_TOYHN8mMifjhLAdqgdyQ2FJp_kWTUNO8j72bt5v8nc04I9fdWpcQM [consultado: 7 de marzo de 2019].

56 XINHUA, “Arabia Saudí descubre enormes reservas de gas en el Mar Rojo”, en Xinhua Español, 8 de marzo de 2019: http://spanish. xinhuanet.com/2019-03/08/c_137876786.htm?fbclid=IwAR3Rsj4X2aRuC2I tcdxpO2mYweJSOnnWmSeqQb7fKxc_QhoKKCA7yQbxUTI [consultado: 8 de marzo de 2019 ].

57 DE YOUNG, Karen, “Saudi Arabia Launches Islamic Military Alliance to Combat Terrorism”, The Washington Post, I5 de diciembre de 2015 https://www.washingtonpost.com/world/national-security/saudi-arabia-launches-islamic-military-alliance-to-combat-terrorism/20I5//2//5/ ad568a I c-a36I-I le5-9c4e-be37f66848bb_story.html?utm_term=.6ccb I Icb I52a [consultado: 7 de marzo de 20I9]. 
noticias de Al Jazeera y el corte de relaciones con Irán, entre otros ${ }^{58}$.

El bloqueo a Qatar, como consecuencia de las ambiciones saudíes al interior del CCG, provocó el acercamiento de este país árabe con Turquía e Irán, países que fueron un apoyo indispensable para que Doha continuara con su dinámica económica y pudiera superar el bloqueo económico de manera paulatina. Esta relación resultó beneficiosa para Ankara y Teherán como países que compiten directamente con Riad en el reacomodo regional del Medio Oriente tras los vacíos de poder generados en las intervenciones militares que se han producido en Siria, Yemen, Iraq y Líbano, al grado de que en octubre de 20 I7Turquía firmó con Sudán una serie de mecanismos de cooperación económica, militar y estratégica, entre los que destacan la construcción de un nuevo aeropuerto en Jartum y una base militar, entre otros ${ }^{59}$.

Con lo anterior, se retoma la parte nodal del argumento central donde el militarismo y el avance de la financiarización económica están estrechamente relacionados pues, la proliferación de más conflictos, o al menos el imaginario de la posible existencia de los mismos, obliga a conectar la reacción de otras potencias regionales a estas alianzas saudíes en el marco de un fuerte y agresivo proceso de capitalismo financiero del que todos los actores, a final de cuentas, salen beneficiados.

\section{Conclusiones}

En este artículo se estudió el papel del proyecto Neom en las dinámicas de financiarización de Arabia Saudí en Oriente Medio y se profundizó en algunas de sus repercusiones regionales. El texto describió a Neom como un proyecto impulsado por élites globales transnacionales cercanas a Mohamemd Bin Salman en el intento de impulsar un modelo económico que deje de depender del petróleo y se incline por uno donde las inversiones extranjeras adquieran un mayor peso. La apuesta de Bin Salman ha sido lo que él llama "la ciudad del futuro", un proyecto totalmente ligado a las dinámicas del capitalismo financiero en el Medio Oriente que implica fuertes inversiones en infraestructura y tecnología, lo cual ha implicado fricciones al interior del reino con otras élites saudíes conectadas con el gran capital como lo ha sido su relación con el Príncipe Alwaleed Bin Talal. Lo anterior se menciona porque es importante resaltar que la existencia de una "súper clase global" no anula la competencia intracapitalista que ha sido núcleo del sistema capitalista desde su nacimiento. La competencia entre las fracciones nacientes transnacionales y las descendientes de grupos dominantes en los Estados a menudo ha sido el telón de fondo en las últimas décadas. Las dinámicas políticas e ideológicas nacionales y la rivalidad entre Bin Salman y actores de la vieja guardia confirman este argumento.

Lo anterior ha tenido consecuencias geopolíticas muy importantes en la zona del Mar Rojo donde se pretende construir Neom, un área de vital importancia geopolítica por las rutas comerciales que van del Canal de Suez hasta el Estrecho de Bab al Mandeb, lo que ayuda a entender diversos conflictos que acontecen en la zona tales como el conflicto irano-saudí en Yemen, la alianza entre Riad con El Cairo y Tel Aviv, y la reacción a dicha alianza entre Qatar, Irán

\footnotetext{
58 ARAB CENTER FOR RESEARCH AND POLICY STUDIES, "The crisis in Gulf Relations: old rivalries, new ambitions", en Arab Center for Research and Policy Studies, Washington, 2017, p.7.

59 MOUBAYED, Sami, “Turkish base in Sudan a problema for Arab Powers", en The Gulf News, 28 de diciembre de 20I7: https://gulfnews.com/ world/mena/turkish-base-in-sudan-a-problem-for-arab-powers-I.2I 48443 [consultado: 7 de marzo de 20I9].
} 
y Turquía.

En este sentido, el texto se enfocó en el estudio de algunos factores locales y regionales para entender la relación que existe entre diversas élites globales trasnacionales saudíes con la generación de conflictos armados y la construcción de más ciudades globales alrededor del mundo, en una relación compleja que se enmarca en el postulado teórico de acumulación por despojo desarrollado por autores como Sassem Robinson y Harvey.

\section{Bibliografía}

ABDUL LATIF JAMEEL, “Arabia Saudí ¿está lista para liderar el desarrollo de ciudades inteligentes en el mundo?, en $A L J, 12$ de agosto de 2018 :

https://www.alj.com/es/perspective/arabia-saudi-esta-lista-para-liderar-el-desarrollo-de-ciudades-inteligentes-en-elmundo/ [consultado: 4 de marzo de 2019].

ADLY,Amr,"Will saudi-egyptian geopolitical partnership push for economic integration? increasing interdependencies", en European University Institute, 2 de febrero de 2019: http://cadmus.eui.eu/bitstream/handle/|8|4/6| |25/ MED_2019_02.pdf?sequence=I\&isAllowed=y [consultado: 5 de marzo de 2019].

AL JAZEERA, "Saudi Crown Prince defends Chine's Right to Fight Terrorism", 23 de febrero de 2019: https://www. aljazeera.com/news/2019/02/saudi-crown-prince-defends-china-fight-terrorism- 190223 I 04647 I 49.html [consultado: 6 de marzo de 2019].

AL-MAANI,Abed Al-Razzak \& ALSHARARI, Saleh. "PearlTrade in the Persian Gulf during the 19th Century", en Asian Culture and History;Vol. 6, No. I; 20I4, pp. 43-52.

ARAB CENTER FOR RESEARCH AND POLICY STUDIES, "The crisis in Gulf Relations: old rivalries, new ambitions”, en Arab Center for Research and Policy Studies, Washington, 2017:

ARAB NEWS, "Saudi Arabia announces creation of Neom Company", en Arab News, 5 de febrero de 2019: http:// www.arabnews.com/node/I44389//saudi-arabia [Consultado: 26 de febrero de 2019].

ARAB NEWS, "Saudi Arabia to start first phase of Neom Project", Arab News. 16 de enero de 2019: http://www. arabnews.com/\%20node\%20/\%201436436\%20/\%20business-economy [Consultado: 27 de febrero de 2019].

ARAB NEWS, Klaus Kleinfeld named adviser to Saudi crown prince, NEOM appoints new CEO, 3 de julio de 20I8: http:// www.arabnews.com/node/I33228I/saudi-arabia [Consultado: 27 de febrero de 2019].

BASSENS, David, BEN Derudder y WITLOX, Frank, "Searching for the Mecca of finance: Islamic financial services and the world city network", en Area,Vol. 42, No. I, 20I0, pp. 35-46.

BELL, Daniel, The Coming of Post-Industrial Society:A Venture in Social Forecasting. Basic Books, New York, 1973.

BUENO, Carmen, "Innovación abierta: de consumidores a productores de valor", en Desacatos, 58, 20I8, pp. 50-69.

CASTELLS, Manuel y HERNÁNDEZ, María, Comunicación y poder,Alianza Editorial, Madrid. 2010.

COX, Robert W., "Social Forces, States and World Orders: Beyond International Relations Theory", en Millennium Journal of International Studies, Junio, I98I, vol. 10, pp. I26-155.

CHANG, Jennifer, "China and Yemen's forgotten War", en United States Institute for Peace. https://www.usip.org/sites/ default/files/2018-0 I/pb24l-china-and-yemens-forgotten-war.pdf [consultado: 5 de marzo de 2019].

DABASHI,Hamid,“The making of a nuclear MBS”, en Al Jazeera, 7 de marzo de 201 9:https://www.aljazeera.com/indepth/ opinion/making-nuclear-mbs- 1 90307 | 35 I | 4759.html?fbclid=IwAR I Hm_TOYHN8mMifjhLAdqgdyQ2FJP_ kWTUNO8j72bt5v8nc04I9fdWpcQM [consultado: 7 de marzo de 2019].

DAVIS, Mike., \& AMOROTO SALIDO, José María, Planeta de ciudades miseria, Foca, Tres Cantos, 2007.

DE YOUNG, Karen, "Saudi Arabia Launches Islamic Military Alliance to Combat Terrorism", The Washington Post, I5 de diciembre de 2015: https://www.washingtonpost.com/world/national-security/saudi-arabia-launchesislamic-military-alliance-to-combat-terrorism/20I5/I2/I5/ad568a I c-a36 I-I le5-9c4e-be37f66848bb_story. html?utm_term=.6ccbl Icb|52a [consultado: 7 de marzo de 2019].

DEFENSE NEWS, “Top ten for 2018”. https://people.defensenews.com/top-100/ [consultado: 4 de marzo de 2019].

DOMINGUEZ, Gabriela, "The Silk Road in the Red Sea”, en Canadian Center for Strategic Studies https://ccss-cces.com/ sections/analysis/2018/05/the-silk-road-in-the-red-sea/ [consultado: 5 de marzo de 2019].

EGAN, Daniel. "Globalization and the invasion of Iraq State power and the enforcement of neoliberalism", en CARLTON-FORD, Steven \& ENDER, Morten (ed.) The Routledge Handbook of War and Society. Iraq and Afghanistan, Routledge, London.

ESPINOSA, Ángeles, "Los saudíes abrazan el cambio sin cuestionar el orden establecido", en El País, 5 de marzo de 2019: https://elpais.com/internacional/2019/03/0I/actualidad//55/436078 66/407.html?id externo rsoc=FB_CC\&fbclid=IwAROvdUdZV23SjTC__dIIWIHleX2W26ctav9EzTv9BE7mjBcMQU6aKA $\bar{K}$ YYOe $\overline{0}$ [consultad̄o: 6 de marzo de 2019].

FANDOS, Nicholas y MAZZETTI, Mark, "House Opens Inquiry Into Proposed U.S. Nuclear Venture in Saudi Arabia", 
en The NewYorkTimes, 19 de febrero de 2019: https:/www.nytimes.com/2019/02/19/us/politics/trump-saudiarabia-nuclear-power.html [consultado: 7 de marzo de 2019].

FIELD, Michael, The Merchants:The Big Business Families of Saudi Arabia and the Gulf States, Overlook Press, Woodstock, N.Y. 1985.

FORBES. “Prince Alwaleed Bin Talal Alsaud”. En Forbes, 20 de marzo de 2017: https://www.forbes.com/profile/princealwaleed-bin-talal-alsaud/\#44c082a4ldbf [consultado: 20 de julio de 2019].

GARCIA, Néstor, “Como investigar la era comunicacional del capitalismo”, en Desacatos, 56, 2018, pp. 90 - I05.

GARDUÑO, Moisés (Coord.) Pensar Palestina desde el Sur Global, UNAM, México, 2017.

GARDUÑO, Moisés. "Medio Oriente: demografía, migración y derechos humanos a siete años de las revoluciones árabes”, en BALLESTEROS, Carlos (coord.). Las Regiones Internacionales, FCPYS-UNAM, México, 2019.

GHAFAR, Adel Abdel,“Educated but unemployed: the challange facing Egypt youth”, en Arab Reform Initiative, octubre 2017.

GULF STATE NEWS, “Succession in the Gulf”, en Gulf State News, http://www.gsn-online.com/succession-in-the-gulfgulf-ruling-families-family-trees, [consultado: 19 de febrero de 2019].

HAJJI ABDULAH ALIREZA \& CO., LTD.“Alireza”. http://www.alireza.com/ [consultado: 20 de febrero de 20I9].

HALL, Richard, "Saudi Arabia: British MPs call for access to detained women activists 'tortured and sexually assaulted' in Saudi prisons", en The Independent, 2 de enero de 2019: https://www.independent.co.uk/news/world/ middle-east/saudi-arabia-women-activists-prison-torture-sexual-assault-ban-driving-dhahban-a870758I . html [consultado: 4 de marzo de 2019].

HAN, Byung-Chul y SARATXAGA Arantzazu, La sociedad del cansancio, Herder, Barcelona, 2012.

HARRIS, Jerry. "Globalization, technology and the transnational capitalist class”, Foresight. I7, 20I 5, pp. I 94-207.

HARVEY, David, A Brief History of Neoliberalism. Oxford University Press, Cary.

HARVEY, David. “The right to the City”, en David Harvey Personal Web Page. https://davidharvey.org/media/ righttothecity.pdf [consultado: 3 de febrero de 2019]

IBRAHIM, Arwa. "What is Sahwa, the Awakening movement under pressure in Saudi?, en Al Jazeera, 5 de junio de 2019: https:/www.aljazeera.com/indepth/features/sahwa-awakening-movement-pressuresaudi- I90603100555782.html [consultado: 6 de julio de 2019].

KELLER, John, "Five U.S defense contractors rank among the world's top 25 cyber security companies", en Military \& Space, https://www.militaryaerospace.com/articles/2018/06/cyber-security-trusted-computing-defensecontractors.html [consultado: 4 de febrero de 2019].

KIRKPATRICK, David. “La purga del príncipe heredero y la transformación radical de Arabia Saudita”, en The New York Times en español, 8 de noviembre de 2017. https://www.nytimes.com/es/20 I7/I l/08/purga-transformacion-arabiasaudita-mohamed-bin-salman/ [consultado: 19 de julio de 2019]

LINS, Gustavo. "El precio de la palabra: la hegemonía del capitalismo electrónico-informático y el googleísmo”, en Desacatos, 58, 2018 , pp. 16-33.

MANJOO, Farhad, "Saudi Money Fuels the Tech Industry It's time to AskWhy”, en The New York Times, 6 de noviembre de 2017: https://www.nytimes.com/20 I 7/I l/06/technology/unsavory-sources-money-fueling-tech.html [consultado: 3 de marzo de 2019].

MIDDLE EAST MONITOR. "Prominent Saudi opposition figure says a revolt is waiting to happen", en Middle East Monitor, 19 de julio de 2019: https:/www.middleeastmonitor.com/201907/9-prominent-saudi-oppositionfigure-says-a-revolt-is-waiting-to-happen/?fbclid=IwAR I nO7BJu6PWj3C9afLyHjjzESfqKK2bfXE2WVDDGUCqFhvq6605Px2-Pw [consultado: 20 de julio de 2019].

MEARSHEIMER, John y WALT Stephen (2006). “The Israel lobby and U.S. foreignpolicy”, en Middle East Policy, XIII (3), p. 45.

MIRTAHERI, Seyed Ahmad, Transnational Capitalism and the Middle East: Understanding the Transnational Elites of the Gulf Cooperation Council, Thesis dissertation, Florida International University, 20 I6, pp. 39-42.

MOUBAYED, Sami, “Turkish base in Sudan a problema for Arab Powers”, en The Gulf News, 28 de diciembre de 2017: https://gulfnews.com/world/mena/turkish-base-in-sudan-a-problem-for-arab-powers- I.2। 48443 [consultado el 7 de marzo de 2019].

NEOM, Foundations. https://www.neom.com/ [Consultado: 26 de febrero de 2019].

NEOM,The members of NEOM. https://www.neom.com/ [Consultado: 27 de febrero de 2019].

OXFAM, "Public Good or Private Wealth, Universal health, education and other public services reduce the gap between rich and poor, and between women and men. Fairer taxation of the wealthiest can help pay for them", Oxfam International, Oxford. p. I2. Disponible en: https://www.oxfamamerica.org/static/media/files/ bp-public-good-or-private-wealth-2I0I I9-en.pdf [consultado: 2 de febrero de 2019].

RASHED, Ingi, “NEOM, a new channelfor Egyptian-Saudi Cooperation”, en Egypt Today, 16 de agosto de 2018: http:// www.egypttoday.com/Article/2/56096/NEOM-A-new-channel-for-Egyptian-Saudi-cooperation [consultado: 5 de marzo de 2019].

REUTERS, “Saudi Arabia to open stock market to foreigners on June 15”, en Reuters 16 de abril de 20I5: https://www. reuters.com/article/saudi-bourse-investment/saudi-arabia-to-open-stock-market-to-foreigners-on-june-I5idUSL5N0XD35P20I504I6 [consultado: 20 de julio de 2019]

ROBEHMED, Natali, "Meet the Richest Peoplein the Middle East", en Forbes, 26 de marzo de 20I4: https://www. forbes.com/sites/natalierobehmed/20 I4/03/26/meet-the-richest-people-in-the-middle-east/\#39b794f2 I bf9 
[consultado: 20 de febrero de 2019].

ROBINSON, William. Latin America and global capitalism: a critical globalization perspective, Johns Hopkins University Press, Baltimore, 2010.

SAID, Abdel Monem y Hussein Ibish, Egypt-GCC Partnership: Bedrock of Regional Security Despite Fissures, The Arab Gulf States Institute in Washington, 12 e diciembre del 2016: https://agsiw.org/egypt-gcc-partnership-bedrockregional-security-despite-fissures/ [consultado: 5 de marzo de 2019].

SASSEN, Saskia, "La ciudad global. Una introducción al concepto y a su historia”, en Brown Journal of World Affairs, vol. I I (2), 1995. pp. 27-43.

SASSEN, Saskia, The Global City: New York, London, Tokyo, Princeton University Press, Oxford, I99I.

SIPRI. “Trends in International Arms Transfers, 2017" Marzo de 20 I8: https://www.sipri.org/sites/default/files/2018-03/ fssipri_at2017_0.pdf [consultado: 4 de marzo de 2019].

SOLER I LECHA, Eduard y Luciano Zaccara, “Arabia Saudí familia, religión, ejército y petróleo”, en BRICHS, Ferran Izquierdo (coord.) Poder y regímenes en el mundo árabe contemporáneo, CIDOB, Barcelona, 2009.

STONE, Brad \& Dean Temple, The Upstarts: How Uber, Airbnb, and the Killer Companies of the New Silicon Valley Are Changing the World, Lilttle Brown and Company, New York, 2017.

THE NEW YORK TIMES, “Egytian PresidentAgrees to Cede 2 Islands to Saudi Arabia”, en The New York Times, 24 de junio de 2017: https://www.nytimes.com/20I7/06/24/world/middleeast/egypt-abdel-fattah-el-sisi-tiransanafir.html [consultado: 3 de marzo de 2019].

THE TIMES OF ISRAEL, “Leaks showEgypt consulted Israel over transfer of Red Sea Islands”, en The Times of Israel, II de febrero de 2017: https://www.timesofisrael.com/leaked-recordings-show-egypt-consulted-israel-overtransfer-of-red-sea-islands/ [consultado: 5 de marzo de 2019].

VISION 2030 KINGDOM OF SAUDI ARABIA. “Foreword”. https://vision2030.gov.sa/en/foreword [consultado: 3 de marzo de 2019].

WINSTANLEY,Asa, "The disappearance of Jamal Khashoggi and the Israel-Saudi Alliance”, en Middle East Monitor, I I de octubre de 2018: https://www.middleeastmonitor.com/2018101 I-the-disappearance-of-jamal-khashoggiand-the-israel-saudi-alliance/ [consultado: 4 de marzo de 2019].

WORLD BANK, "Introduction to Smart cities" https://olc.worldbank.org/content/introduction-smart-cities [consultado: 5 de marzo de 2019].

XINHUA, “Arabia Saudí descubre enormes reservas de gas en el Mar Rojo”, en Xinhua Español, 8 de marzo de 20I9: http://spanish.xinhuanet.com/20I903/08/c_I 37876786.htm?fbclid=IwAR3Rsj4X2aRuC2ItcdxpO2mYweJS OnnWmSeqQb7fKxc_QhoKKCA7yQbxUTI [consultado: 8 de julio de 20I9]. 


\section{RELACIONES INTERNACIONALES}

Revista académica cuatrimestral de publicación electrónica Grupo de Estudios de Relaciones Internacionales (GERI)

Universidad Autónoma de Madrid, España

https://revistas.uam.es/relacionesinternacionales

ISSN 1699 - 3950

f facebook.com/RelacionesInternacionales

3. twitter.com/RRInternacional

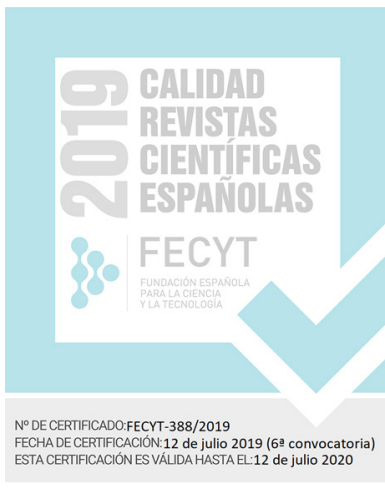

